\title{
Focal partial salvage low-dose-rate brachytherapy for local recurrent prostate cancer after permanent prostate brachytherapy with a review of the literature
}

\author{
Hiroaki Kunogi, MD, PhD', Yoshiaki Wakumoto, MD, PhD², Nanae Yamaguchi, MD', Shigeo Horie, MD, PhD², \\ Keisuke Sasai, MD, PhDl \\ 'Department of Radiation Oncology, ${ }^{2}$ Department of Urology, Juntendo University, Hongo, Bunkyo-ku, Tokyo, Japan
}

\begin{abstract}
Purpose: To investigate the treatment results for focal partial salvage re-implantation against local recurrence after permanent prostate brachytherapy.

Material and methods: Between January 2010 and September 2015, 12 patients were treated with focal partial salvage re-implantation for local recurrence after low-dose-rate brachytherapy using ${ }^{125} \mathrm{I}$ seeds. The focal clinical target volume (F-CTV) was delineated on positive biopsy areas in a mapping biopsy, combining the cold spots on the post-implant dosimetry for initial brachytherapy. The F-CTV was expanded by $3 \mathrm{~mm}$ to create the planning target volume (PTV) as a margin to compensate for uncertainties in image registration and treatment delivery. The prescribed dose to the PTV was 145 Gy. The characteristics and biochemical disease-free survival (BdFS) rates were analyzed. Genitourinary (GU) and gastrointestinal (GI) toxicities were evaluated using the Common Terminology Criteria for Adverse Events version 4.

Results: The median prostate-specific antigen (PSA) level at re-implantation was $4.09 \mathrm{ng} / \mathrm{ml}$ (range: $2.91-8.24 \mathrm{ng} / \mathrm{ml}$ ). The median follow-up time was 56 months (range: 6-74 months). The median $\mathrm{RD}_{2 \mathrm{cc}}$ and $\mathrm{UD}_{10}$ were $63 \mathrm{~Gy}$ and $159 \mathrm{~Gy}$, respectively. The 4-year BdFS rate was 78\%, which included non-responders. Biochemical recurrence occurred in two patients after 7 and 31 months, respectively. The former was treated with hormonal therapy after biochemical failure, and the latter underwent watchful waiting (PSA at the last follow-up of 53 months: $7.3 \mathrm{ng} / \mathrm{ml}$ ) at the patient's request. No patients had grade 3 GU/GI toxicities or died after salvage re-implantation.

Conclusions: The partial salvage low-dose-rate brachytherapy used to treat local recurrence after permanent prostate brachytherapy is well-tolerated, with high biochemical response rates. This treatment can be not only a method to delay chemical castration but also a curative treatment option in cases of local recurrence of prostate carcinoma after seed implantation.
\end{abstract}

Key words: focal therapy, LDR brachytherapy, prostate cancer, re-implantation, salvage.

\section{Purpose}

Permanent prostate brachytherapy using ${ }^{125}$ I for patients with low- or intermediate-risk prostate cancer is an effective treatment option, and can achieve high biochemical progression-free survival [1,2]. However, there are some cases of locoregional recurrence. Currently, there are few recommendations for a relapse of prostate cancer, except for palliative androgen deprivation therapy (ADT). Despite its antineoplastic activity on prostate cancer, the duration of its effectiveness is limited. In retrospective studies, ADT was found to be as- sociated with cardiac sequelae, including coronary heart disease, myocardial infarction (MI), and sudden cardiac death [3]. Considering the site of recurrence in cases of subsequent biochemical relapse, the development of new therapeutic approaches (salvage re-irradiation) will be necessary. Salvage re-irradiation using brachytherapy for local recurrence will be a curative treatment. There are few reports on salvage re-irradiation using ${ }^{125} \mathrm{I}$ permanent prostate brachytherapy for local recurrence after seed implantation.

The purpose of this study was to evaluate the effectiveness of partial salvage re-implantation to develop an 
effective treatment option for local recurrence after permanent prostate brachytherapy.

\section{Material and methods \\ Patients and decision criteria for salvage re-implantation}

Between January 2010 and September 2015, 12 patients were treated with partial salvage ${ }^{125} \mathrm{I}$ brachytherapy for local recurrence after permanent prostate brachytherapy. The 12 patients underwent initial permanent prostatic implantation (monotherapy) using ${ }^{125}$ I radioactive seeds for low- or intermediate-risk prostate carcinoma at our institution between September 2004 and October 2008. Eligibility criteria for focal partial salvage re-implantation were as follows: 1) biochemical failure was defined using the Phoenix criteria (prostate-specific antigen [PSA]-nadir $+2.0 \mathrm{ng} / \mathrm{ml}$ ); 2) a systematic bilateral template-guided prostate/seminal vesicle trans-perineal mapping biopsy with a $5 \mathrm{~mm}$ sampling frame (more than 20 biopsies) was performed for patients with biochemical failure, and locoregional clinical failure was diagnosed by a positive template biopsy; 3) pre-retreatment PSA $<10 \mathrm{ng} / \mathrm{ml}$; 4) no lymph node or distant metastases on computed tomography (CT) or bone scan. A systematic bilateral mapping biopsy was performed according to the procedures described by Bott $e t$ al. [4]. If a biopsy needle placed at the apex could not obtain an adequate sample from the base of the prostate gland, or a cold spot in initial brachytherapy was located in only the apex or base side of the prostate, we divided the biopsy areas into two parts (apex and base sides) and detected the presence of malignancy in each part. As the re-implantation is not considered a standard procedure, locoregional clinical failure and PSA $<10 \mathrm{ng} / \mathrm{ml}$ warranted salvage re-implantation. One patient with a negative biopsy (patient \#1 in Table 1) had a large cold spot (initial $\mathrm{V}_{100}: 58 \%$ ) and long PSA doubling time (15 months), and thus had suspected local recurrent disease, based on reports by Zagars et al. [5] that patients with a PSA doubling time (PSA-DT) exceeding 8 months had only a $7 \%$ metastatic rate and by Zelefsky et al. [6] that the 3-year incidence of distant metastasis for patients with PSA-DT of more than 12 months was 7\%. Written informed consent for treatment was obtained from all patients prior to initial seed implantation and salvage re-implantation. They were also informed about the use of their clinical data. This retrospective study was approved by the ethics board of our institution, and the committee waived the need for re-informed consent.

\section{Focal partial salvage re-implantation planning and procedure}

The focal partial clinical target volume (F-CTV) was delineated on positive biopsy areas in a mapping biopsy, combining the cold spots on the post-implant CTbased dosimetric analysis for initial permanent prostate brachytherapy taking into account microscopic carcinoma in the cold spot with a negative biopsy. Although multi-parametric magnetic resonance imaging (MRI) was performed in 5 of 12 patients before re-implantation, it was impossible to identify the recurrent localization. The F-CTV was expanded by $3 \mathrm{~mm}$ to create the planning target volume (PTV) as a margin to compensate for uncertainties in image registration and treatment delivery, such as movement, and PTV contour was restricted to organs at risk (OARs), such as the rectum and bladder. The dose prescribed to the PTV during intra-operative planning was 145 Gy. Variseed ver. 8.0 software (Varian Medical Systems Inc., Palo Alto, CA, USA) was used for treatment planning (Figure 1). The rectum and urethra were also delineated as OARs on real-time transrectal ultrasonography. Planning criteria for the OAR were according to the following ESTRO/EAU/EORTC recommendations for primary brachytherapy: rectum $D_{2 c c}$ value $\left(R D_{2 c c}\right.$ i.e., the minimum dose in the maximum irradiated $2 \mathrm{cc}$ of the rectum) $<$ the reference prescription dose, and prostatic urethra $\mathrm{D}_{10}$ value $\left(\mathrm{UD}_{10}\right.$; i.e., minimum doses received by $10 \%$ of the urethral volume) $<150 \%$ of the reference prescription dose [7]. To estimate the doses for the entire prostate, the whole prostate was also contoured.

\section{Patient follow-up (PSA value and toxicity)}

Prostate-specific antigen measurements were performed 1 and 6 months post-operatively and subsequently every 3 months. Biochemical failure was defined according to the Phoenix definition [8]. Genitourinary (GU) and gastrointestinal (GI) toxicities were evaluated using the Common Terminology Criteria for Adverse Events version 4.0 (CTCAE-4) [9]. Toxicity was evaluated at baseline, 1, and 6 months post-operatively, and every 6 months thereafter. It was impossible to evaluate erectile dysfunction (ED) for all 12 patients because of incomplete ED status at the time of salvage brachytherapy, although sexual toxicity is often evaluated after radiation therapy [10], and such evaluation is important for re-irradiation studies.

\section{Statistics}

Statistical analyses were performed using SPSS ver. 18 (SPSS Inc., Chicago, IL, USA). Biochemical disease-free survival (BdFS) was estimated according to Kaplan-Meier analysis. $P$-values less than 0.05 were considered to indicate statistical significance.

\section{Results}

\section{Baseline characteristics}

Tables 1 and 2 show the characteristics and summary of each patient. The median PSA nadir after initial permanent prostate brachytherapy was $0.81 \mathrm{ng} / \mathrm{ml}$ (range: $0.37-1.35 \mathrm{ng} / \mathrm{ml}$ ). The median PSA at re-implantation was $4.09 \mathrm{ng} / \mathrm{ml}$ (range: $2.91-8.24 \mathrm{ng} / \mathrm{ml}$ ). The median elapsed time from the initial treatment to salvage treatment was 55 months (range: 34-105 months). The median number of systematic bilateral mapping biopsies per patient was 29.5 (range: $20-36$ ). Three (25\%) patients received neoadjuvant hormonal therapy ranging from 1 to 5 months just before salvage treatment, and no patient received adjuvant hormonal therapy. The median number of seeds per patient in salvage re-implantation was 44 (range: 28-61). The mean activity per seed in salvage re-implantation 


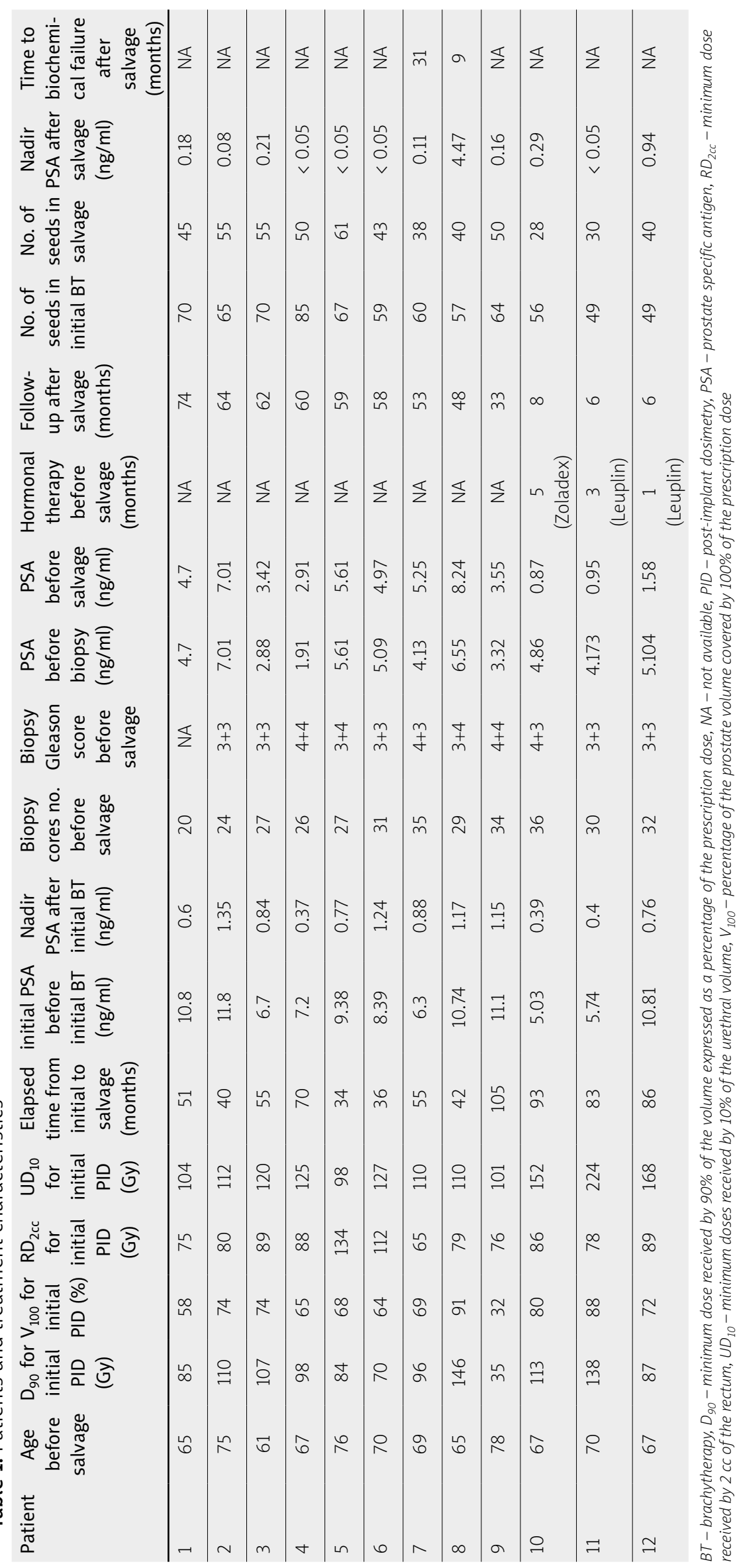




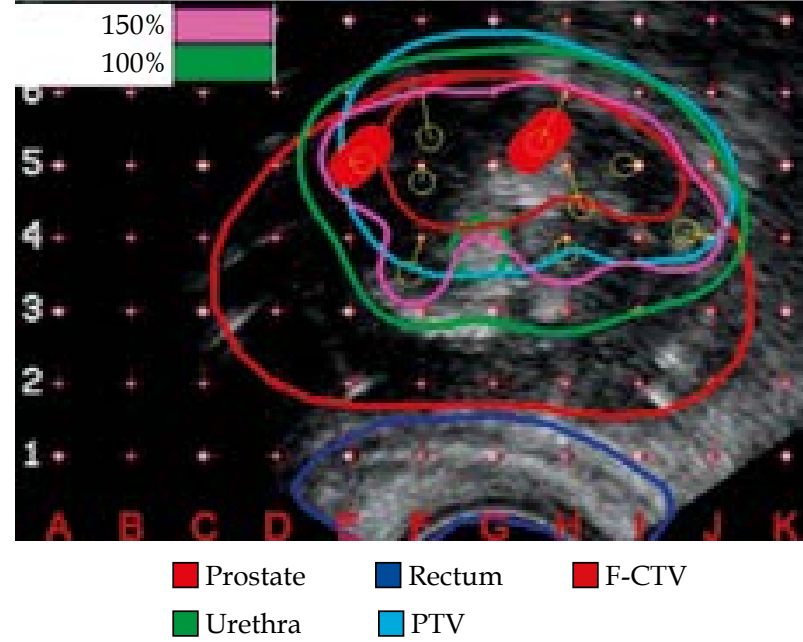

Fig. 1. Example of the dose distribution in intra-operative planning on a representative patient. On the trans-rectal ultrasound image, the focal clinical target volume (dark brown), planning target volume (cyan), prostate (red), urethra (green), and rectum (blue) images are contoured. The dose distributions are shown in light green $(100 \%=145 \mathrm{~Gy})$ and purple $(150 \%=217.5 \mathrm{~Gy})$. The focal clinical target volume received a dose of approximately $150 \%$, and the planning target volume received a dose of approximately $100 \%$. Rectum sparing in the dose distribution is apparent

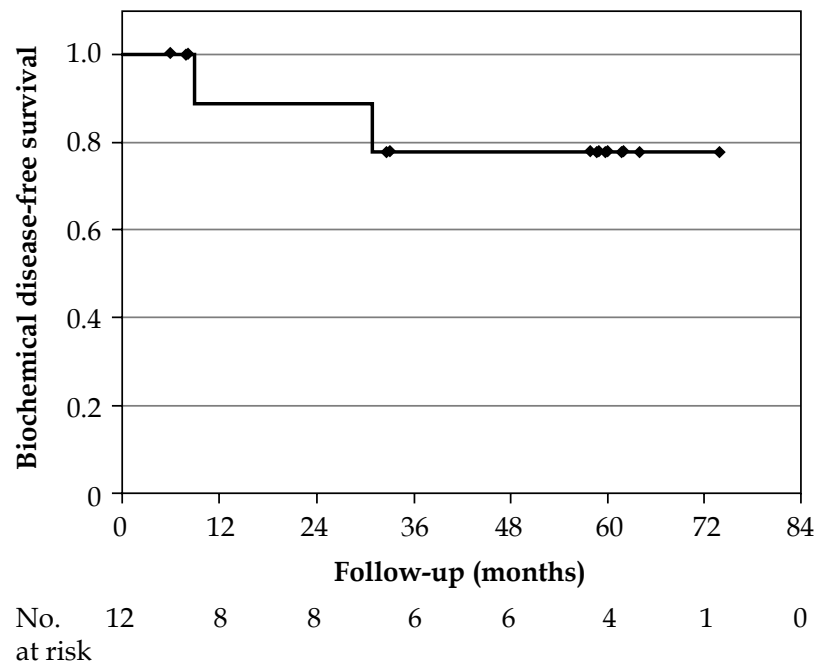

Fig. 2. BdFS estimates for focal partial salvage permanent prostate brachytherapy

was $0.410 \mathrm{U}$ (range: $0.368-0.444 \mathrm{U}$ ). The activity per seed in accordance with the size of the PTV was adequately selected. The median follow-up time was 56 months (range 6-74 months).

\section{Intra-operative dosimetric parameters for F-CTV, $P T V$, rectum, urethra, and prostate in focal partial re-implantation}

The median $D_{90}$ (the minimum dose received by $90 \%$ of the volume expressed as a percentage of the prescription dose) values for F-CTV and PTV were 200 Gy (range:
Table 2. Summary of data for patients at the first treatment

\begin{tabular}{lc} 
Factor & Value (range) \\
\hline PSA at treatment (median) & $8.89 \mathrm{ng} / \mathrm{ml}(5.03-11.8)$ \\
\hline PSA at nadir (median) & $0.81 \mathrm{ng} / \mathrm{ml}(0.37-1.35)$ \\
\hline $\mathrm{D}_{90}$ (median) & $97 \mathrm{~Gy}(35-146)$ \\
\hline $\mathrm{V}_{100}$ (median) & $71 \%(32-91)$ \\
\hline $\mathrm{RD}_{2}$ (median) & $83 \mathrm{~Gy}(65-134)$ \\
\hline $\mathrm{UD}_{10}$ (median) & $116 \%(98-224)$
\end{tabular}

$D_{90}$ - minimum dose received by $90 \%$ of the volume expressed as a percentage of the prescription dose, PSA - prostate specific antigen, $R D_{2}$ - minimum dose received by $2 c c$ of the rectum, $U D_{10}$ - minimum doses received by $10 \%$ of the urethral volume, $V_{100}$ - percentage of the volume covered by $100 \%$ of the prescription dose

145-236) and 165 Gy (range: 129-206), respectively. The median $\mathrm{RD}_{2 \mathrm{cc}}$ and $\mathrm{UD}_{10}$ were 63 Gy (range: $28-89$ ) and 159 Gy (range: 125-198), respectively. The median $D_{90}$ and $V_{100}$ (percentage of the volume covered by $100 \%$ of the prescription dose) values for the prostate were 142 Gy (range: 76-206) and 86\% (range 64-100), respectively.

\section{Biochemical outcomes and survival}

Including non-responders, the Kaplan-Meier 4-year BdFS estimates were $78 \%$ (Figure 2). One patient with a negative biopsy had declined PSA after re-implantation (from 4.7 to $0.23 \mathrm{ng} / \mathrm{ml}$ ), reflecting the implanted coverage of the cold spot areas on post-implant dosimetry for initial brachytherapy. Biochemical failure occurred in two patients $(17 \%$ ) after 7 months (nadir $4.47 \mathrm{ng} / \mathrm{ml}$ ) and 31 months (nadir $0.11 \mathrm{ng} / \mathrm{ml}$ ). The former was treated with ADT after the failure, the latter got a watchful waiting (PSA at the last follow-up of 53 months: $7.3 \mathrm{ng} / \mathrm{ml}$ ) at the patient's request. No patient died after salvage treatment.

\section{Toxicity}

Table 3 summarizes the GU and GI toxicities. The most frequent GU toxicity was grade 1 urinary frequency, often present before salvage re-implantation. Four patients $(33 \%)$ experienced urinary frequency that was managed with medication (grade 2) until the time of the last follow-up. No patient had grade 3 urinary retention. One patient $(8 \%)$ had grade 1 urinary incontinence caused by re-irradiation. No patient experienced grade 2 or more severe urinary incontinence after salvage treatment. One (8\%) experienced radiation cystitis (grade 2), which improved by itself immediately. No patient experienced grade 3 radiation cystitis. No patient had grade 1 radiation proctitis before salvage treatment. After re-implantation, one patient $(8 \%)$ had radiation proctitis (grade 2 ) due to aspirin usage, which improved with no use of aspirin and without laser therapy. No patient experienced grade 3 or higher radiation proctitis.

\section{Discussion}

In this study, focal partial salvage re-implantation against local recurrence of prostate cancer after perma- 
Table 3. Frequencies of GU and GI toxicities

\begin{tabular}{lccccc} 
& Pre-treatment & $\begin{array}{c}12 \text { months } \\
\text { post-treatment }\end{array}$ & $\begin{array}{c}24 \text { months } \\
\text { post-treatment }\end{array}$ & $\begin{array}{c}36 \text { months } \\
\text { post-treatment }\end{array}$ & $\begin{array}{c}48 \text { months } \\
\text { post-treatment }\end{array}$ \\
\hline No. of patients & 12 & 9 & 9 & 8 & 7 \\
\hline GU toxicity & 0 & 4 & 4 & 4 & 3 \\
\hline Grade 2 & 0 & 0 & 0 & 0 & 0 \\
\hline Grade 3 & & & & 0 & 0 \\
\hline GI toxicity & 0 & 0 & 1 & 0 & 0 \\
\hline Grade 2 & 0 & 0 & 0 & &
\end{tabular}

nent prostate brachytherapy provided durable local control. The 4-year BdFS rate after partial salvage re-implantation was $78 \%$. There are few reports with a small number of patients evaluating the effectiveness of partial salvage re-implantation for local recurrence after permanent prostate brachytherapy $[11,12,13]$ (Table 4). Compared with other reports, we illustrated improved tolerability of the treatment with a high PSA control rate at a longer median follow-up time (median: 56 months). Sasaki et al. demonstrated that seven patients underwent salvage partial low dose rate brachytherapy for biopsy-proven, localized prostate cancer recurrence with no significant genitourinary or gastrointestinal toxicity, and the PSA levels remained under control in five patients at a median follow-up of 27 months [11]. In the study, the 2-year BdFS rate after focal salvage re-implantation was not determined but would have been $58 \%$ according to our calculation. Although Peters et al. [12] reported focal salvage permanent prostate brachytherapy in 20 patients, the initial treatments in 13 of these cases were external-beam radiation therapy (EBRT) or intensity-modulated radiation therapy (IMRT). Therefore, the latter finding was fundamentally different from that in our study. Seven prostate cancer cases matched subjects in our study. They demonstrated that the biochemical response and quality of life were acceptable at a median follow-up time of 36 months in 20 patients. However, they did not show the BdFS rates for focal salvage re-implantation alone. Hsu et al. [13] reported that 15 patients underwent MRI-guided focal partial salvage permanent prostate implantation for biopsy-proven localized recurrent prostate cancer after initial low dose rate brachytherapy as definitive monotherapy (13 patients) or combined modality therapy (2 patients). The 3-year BdFS rate after focal partial salvage re-implantation was $71 \%$ in 15 patients at a median follow-up of 23.3 months. However, they did not demonstrate the BdFS rate of re-implantation for local recurrence after initial permanent prostate implant as monotherapy.

The study presented here highlights the possibility to perform re-implantation therapy targeting only the recurrent localized lesion (partial salvage re-implantation) after seed implantation, not only to prevent or postpone the use of ADT but also to cure prostate carcinomas. Androgen deprivation therapy is the mainstay of treatment for a relapse of prostate cancer after seed implantation. The impact of immediate ADT on specific survival for prostate cancer in PSA relapse is unclear except in patients with rapidly progressing disease [14]. Some retrospective reports with a small number of patients have shown the feasibility of salvage local irradiation for localized persistent prostate cancer (LDR brachytherapy after EBRT [15]/radical prostatectomy [16], and stereotactic radiotherapy after LDR brachytherapy [17]).

Permanent prostate brachytherapy sometimes causes insufficient dose coverage due to the technique. Immediate re-implantation as a salvage treatment is recommended in such cases [18]. We did not re-implant immediately for the under-dosed area and carefully observed the PSA values. Indeed, in 11 of 12 patients in our study, the $D_{90}$ for the entire prostate could not sufficiently ensure the prescription dose (145 Gy). However, the median initial PSA before initial brachytherapy and the median nadir PSA after initial brachytherapy were 8.9 and $0.8 \mathrm{ng} / \mathrm{ml}$, respectively (maximum nadir PSA: $1.35 \mathrm{ng} / \mathrm{ml}$ ). The largest tumor focus within the prostate (called the index lesion) [19] seemed to be irradiated. Consequently, there was no need to re-implant right after the initial implantation.

The Gleason score was increased at recurrence in 6 of 12 patients $(50 \%)$ compared with that at the initial brachytherapy in this study. The high score indicates that it is difficult to achieve durable disease control after salvage treatment. However, we did demonstrate a high durable disease control rate (4-year BdFS rate: $78 \%$ ). Due to histological changes after radiation therapy, it may not always be accurate for recurrent prostate carcinoma to be evaluated for malignancy using the Gleason system according to recommendations of both the College of American Pathologists and World Health Organization [20].

Salvage re-irradiation using high-dose-rate brachytherapy can also be a treatment option. Chen et al. [21] reported that the 5-year biochemical control was $51 \%$ at a median follow-up time of 59.6 months after salvage high dose rate brachytherapy, and late grade $3 \mathrm{GU} / \mathrm{GI}$ and late grade $2 \mathrm{GU} / \mathrm{GI}$ toxicities were observed in $2 \%$, $0 \%, 54 \%$, and $4 \%$, respectively. It is difficult to say which brachytherapy is superior to others at this time.

Seed migration may be critical for partial salvage LDR brachytherapy. Wang et al. reported that it had no im- 


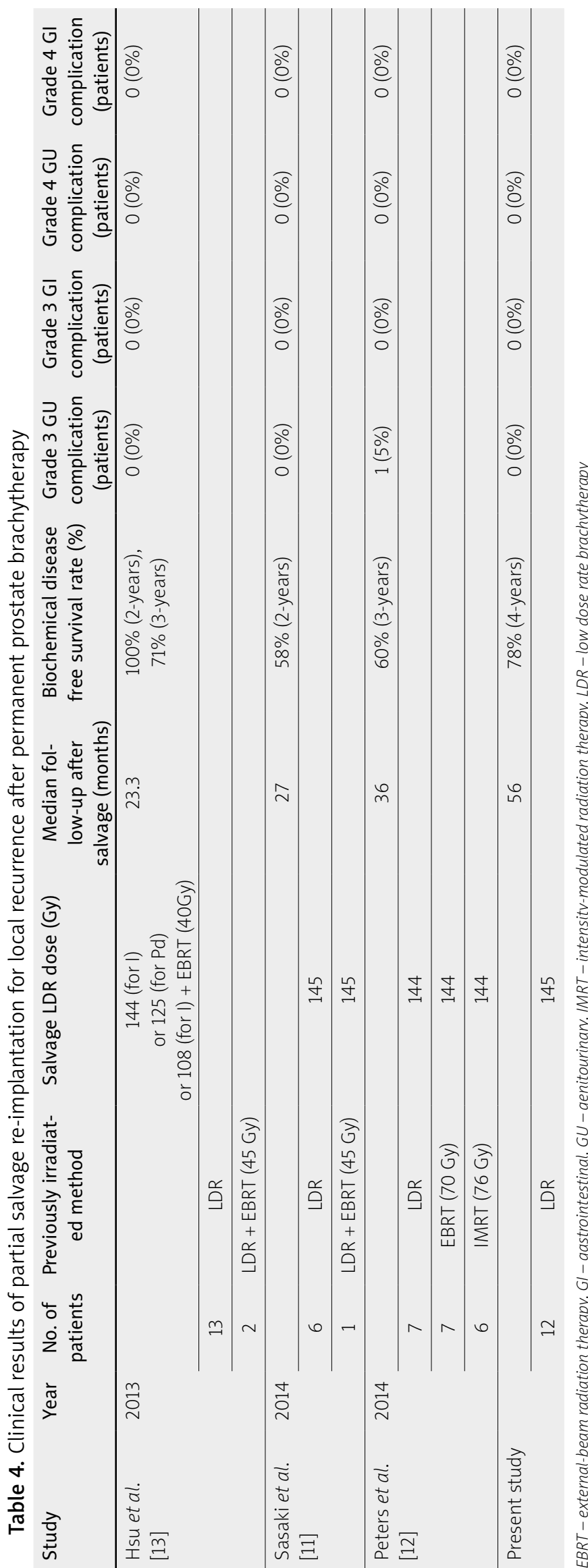

pact on the implant quality of initial whole prostate LDR brachytherapy [22]. However, in focal partial re-implantation, it is necessary to avoid seed migration to maintain implant quality.

The optimized tumor control dose to the target is undetermined at present. In this study, the dose prescribed to the PTV during intra-operative planning was $145 \mathrm{~Gy}$. Sasaki et al. designed salvage partial low dose rate brachytherapy delivering 144 Gy to positive biopsy sites, corresponding to cold spot areas at the initial low dose rate brachytherapy [11]. Peters et al. reported that a dose of $>144$ Gy was prescribed to the target area and was delineated on MRI combining biopsy results [12]. Hsu et al. reported that the prescription dose for implants was $144 \mathrm{~Gy}$ for ${ }^{125} \mathrm{I}, 125 \mathrm{~Gy}$ for ${ }^{103} \mathrm{Pd}$, and $108 \mathrm{~Gy}$ for ${ }^{125} \mathrm{I}$ with IMRT (40 Gy) [13]. Future investigations for prescription dose recommendations are required to estimate salvage re-implantation as a curable treatment.

The recommended CTV delineation and PTV margin size is also unclear at present. Langley et al. [23] reported that the F-CTV is delineated as the gross visible or clinically demonstrable location and the extent of the targeted cancer plus clinically insignificant disease; it was expanded to create the focal PTV to compensate for uncertainties in image registration and treatment delivery, such as movement, and the focal PTV contours would be restricted to OAR contours such as those of the urethra and rectum. Groenendaal et al. [24] reported that the application of a margin of 2 voxels (voxel size $2.5 \times 2.5 \times 2.5 \mathrm{~mm}^{3}$; $5 \mathrm{~mm}$ margin) to an MR-based tumor delineation in five patients with biopsy-proven prostate cancer (PSA: 14-29 ng/ml; pathological Gleason: 6-8; pathological T stage: T2a-T3a) enables the coverage of approximately $85-100 \%$ of the tumor. Sasaki et al. [11] reported that the clinical target volume (CTV) was manually delineated as a region of local recurrence with biopsy positivity and initial under-dosage according to MRI/initial CT dosimetry, and the PTV included the CTV plus a $5 \mathrm{~mm}$ margin $(5 \mathrm{~mm}$ posterior margin). Peters et al. [12] reported that the treatment margins were expanded up to half of the prostate to account for uncertainties in the definition and delineation of the gross tumor volume (based on T2-weighted magnetic resonance imaging [T2W-MRI]), combining biopsy results and multi-parametric MRI and the uncertainty in matching of the MRI and ultrasound images. Hsu et al. [13] demonstrated MRI-guided focal partial salvage permanent prostate implantation for local recurrent prostate cancer after initial implantation, and they defined the CTV as defined as an area of local recurrence with initial under-dosage. In the current study, F-CTV was not delineated on imaging modalities such as MRI but rather on positive biopsy areas in a $5 \mathrm{~mm}$ mapping biopsy, combining the cold spots on post-implant dosimetry and taking into account microscopic carcinoma in the cold spot with a negative biopsy. We performed MRI in 5 of 12 cases to delineate the recurrent gross tumor; however, it was impossible to identify the location of the tumor in 5 cases. If the needle biopsy did not sufficiently cover the apex to the base of the prostate gland or the cold spot in initial brachytherapy located in only the apex or base 
side of the prostate, we divided the biopsy areas into two parts (apex and base sides) and detected the presence of malignancy in each part. The F-CTV will not necessarily include the cold spot with a negative biopsy, thus, it is difficult to conclude that our F-CTV is adequate. Regarding the PTV margin size, the F-CTV was expanded by $3 \mathrm{~mm}$ to create the PTV as a margin to compensate for uncertainties in image registration and treatment delivery. In the re-implantation study, a $3 \mathrm{~mm}$ margin to both the positive biopsy areas and cold spots may also be a practical first step. Without a systematic comparison of margin distances, we cannot conclude that $3 \mathrm{~mm}$ is an adequate distance.

More accurate diagnostic imaging can improve the treatment effect for better disease control. Biochemical progression is due to either local recurrence or distant metastasis, and the PSA doubling time was identified as a predictive factor of distant metastases $[5,6,25]$. Peter et al. and Sasaki et al. demonstrated that, due to less time to biochemical recurrence/a shorter PSA doubling time, some patients failed to reflect probable (micro) metastatic disease at the time of focal salvage $[11,12]$. In the present study, one patient who had a shorter PSA doubling time at the time point of partial salvage had PSA progression after salvage treatment. A shorter PSA doubling time may imply the existence of regional or distant recurrence, even if locoregional clinical failure was diagnosed by a positive biopsy, and no lymph node or distant metastases were diagnosed on pelvic-CT or bone scan.

This study analyzed only a small number of patients retrospectively. Partial salvage re-implantation for patients with biochemical failure after permanent brachytherapy was effective and safe. However, it is difficult to draw a conclusive result from such a population. A large clinical trial regarding such a treatment may be warranted. In that case, careful follow-up after re-irradiation is important, including prevention of constipation-reducing rectal toxicity [26], because a previous prospective phase II study on whole prostate salvage LDR brachytherapy after EBRT or brachytherapy reported $30 \%$ grade $3-4$ GI toxicities and $13 \%$ grade $3-4$ GU toxicities [27].

\section{Conclusions}

Partial salvage low dose rate brachytherapy for local recurrence after permanent prostate brachytherapy is well tolerated, with high biochemical response rates. This treatment can be not only a method to delay chemical castration but also a curative treatment option in cases of local recurrence of prostate carcinoma after seed implantation.

\section{Disclosure}

Authors report no conflict of interest.

\section{References}

1. Grimm P, Billiet I, Bostwick D et al. Comparative analysis of prostate-specific antigen free survival outcomes for patients with low, intermediate and high risk prostate cancer treat- ment by radical therapy. Results from the Prostate Cancer Results Study Group. BJU Int 2012; 109 Suppl 1: 22-29.

2. Guinot JL, Ricós JV, Tortajada MI et al. Comparison of permanent (125) I seeds implants with two different techniques in 500 cases of prostate cancer. J Contemp Brachytherapy 2015; 7: 258-264.

3. Keating NL, O'Malley AJ, Smith MR. Diabetes and cardiovascular disease during androgen deprivation therapy for prostate cancer. J Clin Oncol 2006; 24: 4448-4456.

4. Bott SR, Henderson A, McLarty E et al. A brachytherapy template approach to standardize saturation prostatic biopsy. BJU Int 2004; 93: 629-630.

5. Zagars GK, Pollack A. Kinetics of serum prostate-specific antigen after external beam radiation for clinically localized prostate cancer. Radiother Oncol 1997; 44: 213-221.

6. Zelefsky MJ, Ben-Porat L, Scher HI et al. Outcome predictors for the increasing PSA state after definitive external beam radiotherapy for prostate cancer. J Clin Oncol 2005; 23: 826-831.

7. Salembier C, Lavagnini P, Nickers $P$ et al. Tumour and target volumes in permanent prostate brachytherapy: a supplement to the ESTRO/EAU/EORTC recommendations on prostate brachytherapy. Radiother Oncol 2007; 83: 3-10.

8. Roach M 3rd, Hanks G, Thames H Jr et al. Defining biochemical failure following radiotherapy with or without hormonal therapy in men with clinically localized prostate cancer: recommendations of the RTOG-ASTRO Phoenix Consensus Conference. Int J Radiat Oncol Biol Phys 2006; 65: 965-974.

9. NCI. CTCAE. Available from: http://evs.nci.nih.gov/ftp1/ CTCAE/About.html. Accessed May, 17, 2010.

10. Olsson CE, Alsadius D, Pettersson $N$ et al. Patient-reported sexual toxicity after radiation therapy in long-term prostate cancer survivors. Br J Cancer 2015; 113: 802-808.

11. Sasaki H, Kido M, Miki K et al. Salvage partial brachytherapy for prostate cancer recurrence after primary brachytherapy. Int J Urol 2014; 21: 572-577.

12. Peters M, Maenhout M, van der Voort van Zyp JR et al. Focal salvage iodine-125 brachytherapy for prostate cancer recurrences after primary radiotherapy: a retrospective study regarding toxicity, biochemical outcome and quality of life. Radiother Oncol 2014; 112: 77-82.

13. Hsu CC, Hsu H, Pickett B et al. Feasibility of MR imaging/ MR spectroscopy-planned focal partial salvage permanent prostate implant (PPI) for localized recurrence after initial PPI for prostate cancer. Int J Radiat Oncol Biol Phys 2013; 85: 370-377.

14. Studer UE, Whelan P, Wimpissinger F et al. Differences in time to disease progression do not predict for cancer-specific survival in patients receiving immediate or deferred androgen-deprivation therapy for prostate cancer: final results of EORTC randomized trial 30891 with 12 years of follow-up. Eur Urol 2014; 66: 829-838.

15. Chang L, Buyyounouski MK. Salvage low-dose-rate (125)I partial prostate brachytherapy after dose-escalated external beam radiotherapy. J Contemp Brachytherapy 2014; 6: 304-310.

16. Kumar AM, Smith KL, Reddy CA et al. Safety and efficacy of salvage low-dose-rate brachytherapy for prostate bed recurrences following radical prostatectomy. J Contemp Brachytherapy 2015; 7: 241-246.

17. Eppinga W, Vijverberg $\mathrm{P}$, Moerland $\mathrm{R}$ et al. Perineal recurrence of prostate cancer six years after trans-perineal brachytherapy. J Contemp Brachytherapy 2015; 6: 386-388.

18. Putora PM, Plasswilm L, Seelentag W et al. Re-implantation after insufficient primary 125 -i permanent prostate brachytherapy. Radiat Oncol 2013; 8: 194.

19. Ahmed HU. The index lesion and the origin of prostate cancer. N Engl J Med 2009; 361: 1704-1706. 
20. Bostwick DG, Foster CS. Predictive factors in prostate cancer: current concepts from the 1999 College of American Pathologists Conference on Solid Tumor Prognostic Factors and the 1999 World Health Organization Second International Consultation on Prostate Cancer. Semin Urol Oncol 1999; 17: 222-272.

21. Chen $\mathrm{CP}$, Weinberg V, Shinohara $\mathrm{K}$ et al. Salvage HDR brachytherapy for recurrent prostate cancer after previous definitive radiation therapy: 5-year outcomes. Int J Radiat Oncol Biol Phys 2013; 86: 324-329.

22. Wang Y, Nasser NJ, Borg J et al. Evaluation of the dosimetric impact of loss and displacement of seeds in prostate low-dose-rate brachytherapy. J Contemp Brachytherapy 2015; 7: 203-210.

23. Langley S, Ahmed HU, Al-Qaisieh B et al. Report of a consensus meeting on focal low dose rate brachytherapy for prostate cancer. BJU Int 2012; 109: 7-16.

24. Groenendaal G, Moman MR, Korporaal JG et al. Validation of functional imaging with pathology for tumor delineation in the prostate. Radiother Oncol 2010; 94: 145-150.

25. Stock RG, Cesaretti JA, Unger P et al. Distant and local recurrence in patients with biochemical failure after prostate brachytherapy. Brachytherapy 2008; 7: 217-222.

26. Otón LF, Dolado MC, Núñez EJ et al. Effect of constipation on dosimetry after permanent seed brachytherapy for prostate cancer. J Contemp Brachytherapy 2015; 7: 247-251.

27. Nguyen PL, Chen MH, D'Amico AV et al. Magnetic resonance image-guided salvage brachytherapy after radiation in select men who initially presented with favorable-risk prostate cancer: a prospective phase 2 study. Cancer 2007; 110: 1485-1492. 